\title{
Schilddrüsenunterfunktion
}

\section{Jodversorgung in Deutschland nicht ausreichend}

Deutschland ist kein Jodmangelgebiet, allerdings ist die Jodversorgung alles andere als optimal. Die Ergebnisse der bundesweiten Studie zur Gesundheit Erwachsener in Deutschland (DEGS) deuten sogar auf eine Verschlechterung der Versorgung hin. Dies war der Anlass für Wissenschaftler der Universität Bonn

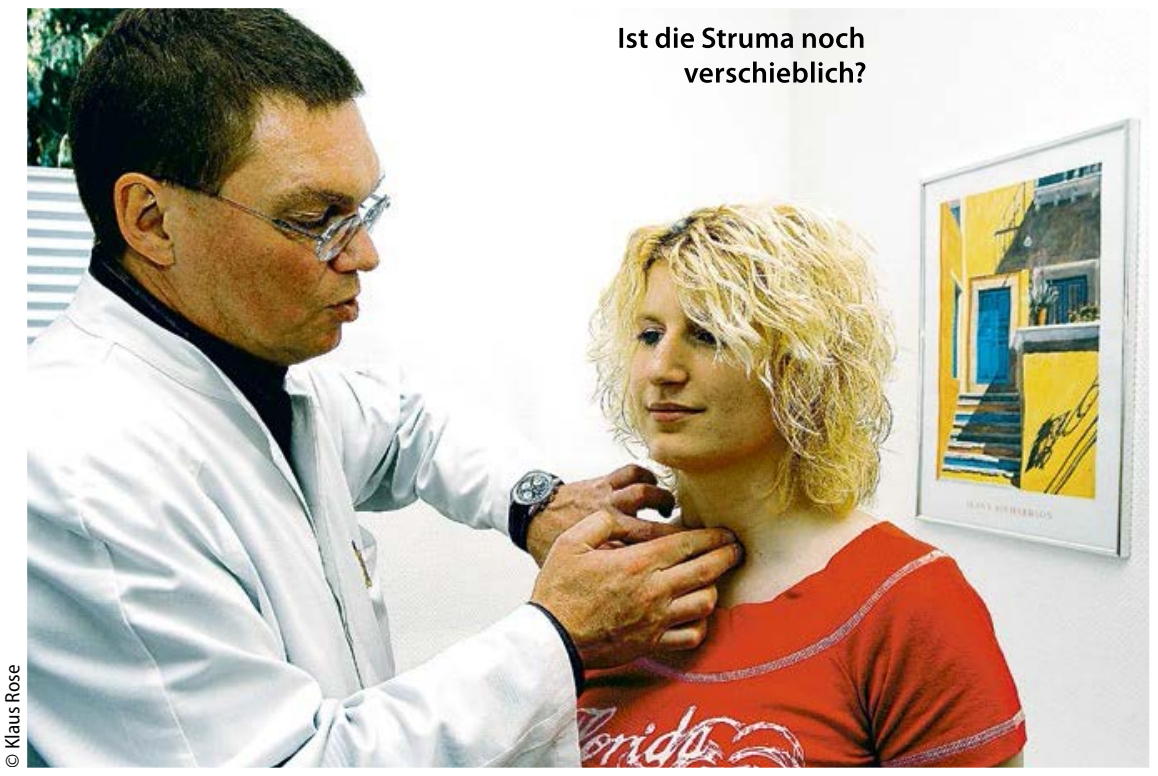

und des Robert-Koch-Instituts, den Versorgungsstatus sowie Auswirkungen des Hydratationsstatus auf die Urin-Jodkonzentration (UIC) näher zu untersuchen.

Die Bestimmung der Jodausscheidung im Urin ist die empfohlene Methode, um den Jodversorgungsstatus einer Population zu beurteilen. Das Ergebnis:

\section{die Struma noch}

Die mediane UIC betrug

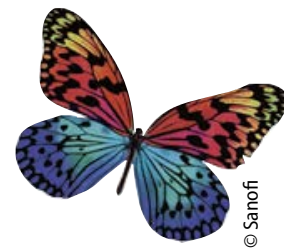

bei Männern $69 \mu \mathrm{g} / \mathrm{l}$ und bei Frauen

$54 \mu \mathrm{g} / \mathrm{l}$. Dies liegt deutlich unter dem WHO-Grenzwert für eine ausreichende Jodversorgung $(100 \mu \mathrm{g} / \mathrm{l})$. Daher fordern die Autoren der Untersuchung, Maß nahmen zur Jodprophylaxe verstärkt zu fördern.

Die Autoren warnen ferner davor, das Urinvolumen bei der Betrachtung der Jodkonzentration außen vor zu lassen. Dessen Einfluss sei naturgemäß sehr groß, was zu einer erheblichen Fehleinschätzung der Jodversorgung einer Population führen könne. Hier ist unter anderem entscheidend, wie konzentriert oder verdünnt der Urin ist bzw. wie hoch das mittlere Urinvolumen pro Tag ist. Diese Parameter hängen eng mit dem Hydratationsstatus der Testpersonen zusammen.

Übrigens gibt es auch im Jahr 2016 wieder eine Schilddrüsenwoche. Sie findet vom 25. bis 29. April statt. Mehr Informationen dazu gibt es online unter www.infoline-schilddruese.de.

Dr. Christine Ott

- Johner SA et al. Eur J Nutr 2015, online 2. Juni

\section{Diabetes mellitus}

\section{Online-Portal erleichtert Einstieg in basal unterstützte orale Therapie}

Den Beginn einer Insulintherapie empfinden viele Patienten als große Belastung. Deshalb gibt es „Starthilfe“ zum neuen Insulin glargin U100 Abasaglar ${ }^{\circledR}$. Klar und übersichtlich gestaltete Materialien sowie eine Website, die auch kurze Anwendungsvideos anbietet, sollen dem Patienten einen guten Start in die Basalinsulin-Therapie ermöglichen.

Die Website richtet sich aber auch an Ärzte und Diabetesberater. Diese finden in einem speziellen Bereich praktische Tipps zum Umgang mit den Patienten. Für Ärzte gibt es in einem geschützten Bereich Informationen zu den Zulassungsstudien sowie unter der Rubrik
„Erstattung“ eine Übersicht, die zeigt, dass das Biosimilar Abasaglar ${ }^{\circledast}$ von nahezu allen gesetzlichen Krankenkassen erstattet wird.

\section{Mehrere Hilfestellungen für Diabetespatienten}

Doch nicht nur Ärzte und Diabetesberater, auch Patienten soll die neue Website startklar machen. Die können die in der Einstellungsmappe enthaltenen Servicematerialien einfach herunterladen: eine Patientenbroschüre zur Basalinsulin-Therapie, eine Kurzanleitung für die Anwendung des Abasaglar ${ }^{\circledast}$ KwikPen $^{\mathrm{TM}}$ und ein Blutzucker-Tagebuch. Zudem sind kurze Videos online, die die Anwendung der neuen Insulin-glarginOption von Lilly und Boehringer Ingelheim einprägsam und leicht verständlich erläutern.

Abasaglar ${ }^{\circledast}$ ist das erste Insulin-Biosimilar, das in Europa zugelassen wurde. In Deutschland ist die neue Option im Bereich Insulin glargin U100 seit September auf dem Markt. Sie ist zur Behandlung von Erwachsenen und Kindern ab eine Alter von zwei Jahren mit Diabetes mellitus Typ 1 oder Typ 2 zugelassen.

$$
\text { Red. }
$$

- Nach Informationen von Boehringer Ingelheim und Lilly 